\title{
Rotating Fluids with Self-Gravitation in Bounded Domains
}

\author{
TAO LUO \& JOEL SMOLLER
}

Communicated by P. H. RABINOWITZ

\begin{abstract}
In this paper, we study the steady solutions of Euler-Poisson equations in bounded domains with prescribed angular velocity. This models a rotating Newtonian star consisting of a compressible perfect fluid with given equation of state $P=e^{S} \rho^{\gamma}$. When the domain is a ball and the angular velocity is constant, we obtain both existence and non-existence theorems, depending on the adiabatic gas constant $\gamma$. In addition we obtain some interesting properties of the solutions; e.g., monotonicity of the radius of the star with both angular velocity and central density. We also prove that the radius of a rotating spherically symmetric star, with given constant angular velocity and constant entropy, is uniformly bounded independent of the central density. This is physically striking and in sharp contrast to the case of the non-rotating star. For general domains and variable angular velocities, both an existence result for the isentropic equations of state and non-existence result for the non-isentropic equation of state are also obtained.
\end{abstract}

\section{Introduction}

The purpose of this paper is to understand the structure of a rotating star. In the theory of General Relativity, for a non-rotating star consisting of a perfect fluid, the exterior and interior solutions are very well understood. The exterior solution of the star, that is, the gravitational field (space time metric) outside the star, is given by the well-known Schwarzschild solution, and the interior solution, which matches the exterior Schwarzschild solution on the boundary of the star, is obtained by solving the Tolman-Oppenheimer-Volkoff equations [18]. For a rotating star, the exterior solution of the gravitational field is the celebrated Kerr solution [1]. However, no corresponding interior solution of Einstein's equation is known which matches the Kerr solution at the boundary of the rotating star. Actually, even in the Newtonian case, unlike the case of the non-rotating star which has been extensively studied 
by Ritter, Lane, Emden, Kelvin and Chandrasekhar; (see [5]; and also [8] and [13] for more recent results in the case of a non-rotating star), there have been very few results on the rotating star if the star consists of a compressible perfect fluid. This paper is devoted to the investigation of the steady solutions of a compressible perfect fluid rotating star in the Newtonian case with prescribed angular velocity, in a bounded domain, and with zero density on the boundary chosen to match the exterior of the star.

The motion of the compressible perfect fluid with self-gravitation is modelled by the following Euler-Poisson equations; cf. [5],

$$
\begin{aligned}
\rho_{t}+\operatorname{div}_{x}(\rho \mathbf{v}) & =0, \\
\rho v_{t}+(\rho \mathbf{v} \cdot \nabla) \mathbf{v}+\nabla P+\rho \nabla \Phi & =0 \\
S_{t}+\mathbf{v} \cdot \nabla S & =0, \\
\Delta \Phi & =4 \pi G \rho,
\end{aligned}
$$

where $\rho, \mathbf{v}, P, S$ and $\Phi$ denote the density, velocity, pressure, entropy and gravitational potential, respectively. Here $(t, x) \in \mathbf{R}^{+} \times \mathbf{R}^{\mathbf{3}}$ denotes the time and space variables and $x=\left(x_{1}, x_{2}, x_{3}\right)$. For simplicity, we assume the pressure satisfies the following equation of state:

$$
P=P(\rho, S)=e^{S} \rho^{\gamma},
$$

where $\gamma>1$ is the adiabatic exponent.

Suppose the star rotates about the $x_{3}$-axis; we are interested in finding an axisymmetric solution $(\rho, \mathbf{v}, S, \Phi)(x, t)=(\rho, \mathbf{v}, S, \Phi)\left(\eta(x), x_{3}, t\right)$ of (1.1) with prescribed time-independent angular velocity $\Omega(\eta)$, where

$$
\eta=\eta(x)=\sqrt{x_{1}^{2}+x_{2}^{2}} .
$$

In this case the velocity field is given by $\mathbf{v}=\left(-x_{2} \Omega(\eta), x_{1} \Omega(\eta), 0\right)$, and thus $\operatorname{div}_{x}(\rho \mathbf{v})=0$ and $\mathbf{v} \cdot \nabla S=0$. By $(1.1)_{1}$ and $(1.1)_{3}, \rho_{t}=0$ and $S_{t}=0$. Thus, the solution $(\rho, \mathbf{v}, S, \Phi)$ of (1.1) is time-independent and satisfies the following system of equations:

$$
\begin{aligned}
\nabla P(\rho, S)+\rho \nabla \Phi-\rho \nabla J(\eta) & =0, \\
\Delta \Phi & =4 \pi G \rho,
\end{aligned}
$$

where

$$
J(\eta)=\int_{0}^{\eta} s \Omega^{2}(s) d s
$$

and the entropy $S$ is a given bounded $C^{1}$ function.

In [2], for an isentropic fluid, i.e., the entropy $S=$ constant (without loss of generality, $\mathrm{S}$ is assumed to be zero in this case), AUCHMUTY \& BEALS considered system (1.3) in all of $R^{3}$, with prescribed total mass $M$, and gravitational potential $\Phi$ given by

$$
\Phi(x)=-G \int_{R^{3}} \frac{\rho(y)}{|x-y|} d y=:-B \rho(x) .
$$


The problem then reduces to finding the solution of the equation

$$
\nabla\left(\frac{\gamma \rho^{\gamma-1}}{\gamma-1}-B \rho-J(\eta)\right)(x)=0,
$$

where $\rho>0$. They formulated this as a variational problem; namely, minimize

$$
E(\rho)=\int_{R^{3}}\left(\frac{\rho^{\gamma}}{\gamma-1}-\frac{1}{2} \rho \cdot B \rho-\rho J(\eta)\right)(x) d x
$$

in the class

$$
W_{M}=\left\{\rho \geq 0: \int_{R^{3}} \rho(x) d x=M\right\} .
$$

By assuming that the angular velocity $\Omega$ satisfies the decay properties

$$
\begin{aligned}
& J(+\infty)<+\infty, J \in C^{1}[0,+\infty), \\
& \eta(J(+\infty)-J(\eta)) \rightarrow 0 \text { as } \eta \rightarrow+\infty,
\end{aligned}
$$

and that the adiabatic exponent $\gamma$ satisfies

$$
\gamma>4 / 3
$$

Auchmuty and Beals proved the existence of a minimizer of the functional $E(\rho)$ in the class of functions (1.7). Moreover, this minimizer has compact support and satisfies equation (1.5) wherever it is positive. The shape of the free boundary which separates the vacuum and fluid was investigated in [3] for the AuchmutyBeals solutions. The case of an isentropic uniformly rotating star, (i.e., the angular velocity $\Omega$ is constant), was discussed by $L i$ in ([11]); he proved the existence of a minimizer of the functional (1.6) in the class $W_{M}$, under the assumption $\gamma>4 / 3$. The diameter of the support of the density $\rho$ was studied in [6] for the solution obtained in [11]. In the proof of the above results, the prescribed total mass serves as a constraint on these variational problems, and without this constraint, it is not clear that the minimizer of $E(\rho)$ exists. In [2], the angular velocity is prescribed in the entire space $R^{3}$ (even in the vacuum region), and is assumed to satisfy the decay property (1.8). In [2] and [11], $\gamma$ is required to be greater than $4 / 3$, so a natural question to ask is: What happens when $1<\gamma \leq 4 / 3$ ? Another issue is that all of the above-mentioned results are for isentropic fluids, so we can also ask: What happens if the fluid is non-isentropic? The purpose of this paper is to address these, and other issues.

\section{Statement of results}

We are interested in the solution of (1.3) in a bounded domain $D$ in $R^{3}$, where $\rho(x)>0$ for $x \in D$ and $\rho(x)=0$ if $x \in \partial D$. From the first equation in system (1.3), we have

$$
\frac{1}{\rho} \nabla P=\nabla(J-\Phi)
$$


for $x \in D$. Thus, by the second equation of (1.3), we obtain

$$
\operatorname{div}\left(\frac{1}{\rho} \nabla P\right)=\Delta(J-\Phi)=\Delta J-4 \pi G \rho
$$

for $x \in D$. Set

$$
w=\frac{\gamma}{\gamma-1}\left(e^{S / \gamma} \rho\right)^{\gamma-1}
$$

Then it is easy to verify, using (1.2), that

$$
\frac{1}{\rho} \nabla P=e^{S / \gamma} \nabla w .
$$

Substituting this in (2.2), we obtain the elliptic equation

$$
\operatorname{div}\left(e^{\alpha S} \nabla w\right)+K e^{-\alpha S} w^{q}-2 \Omega(\eta)\left(\Omega(\eta)+\eta \Omega^{\prime}(\eta)\right)=0,
$$

where

$$
q=\frac{1}{\gamma-1}, \quad \alpha=\frac{1}{\gamma}
$$

$K=4 \pi G\left(\frac{\gamma-1}{\gamma}\right)^{\frac{1}{\gamma-1}}$, and for simplicity, we can normalize $K$ to make $K=1$. We seek solutions of (2.5) satisfying

$$
\rho(x)>0, \quad x \in D, \quad \rho(x)=0, \quad x \in \partial D,
$$

or equivalently

$$
w(x)>0, \quad x \in D, \quad w(x)=0, \quad x \in \partial D .
$$

In this paper, we only consider the case when

$$
1<\gamma<2
$$

because when $\gamma>2,0<q<1$, and equation (2.5) becomes sublinear, and this situation was studied completely in [17]. Moreover, equation (2.5) is linear if $\gamma=2$, and there is a complete theory for linear elliptic equations (cf. [10]).

We first consider the case when the angular velocity $\Omega(\eta)=\Omega=$ constant, the entropy $S(x)$ is spherically symmetric (i.e., $S(x)=S(r), r=|x|=\sqrt{x_{1}^{2}+x_{2}^{2}+x_{3}^{2}}$ ), and the domain $D$ is a ball $B_{R}(0)$. In this case, we look for the spherically symmetric solutions of problem (2.5) and (2.8), i.e, $w(x)=w(r), r=|x|=\sqrt{x_{1}^{2}+x_{2}^{2}+x_{3}^{2}}$. Then $w(r)$ satisfies the equation

$$
w^{\prime \prime}(r)+\left(\frac{2}{r}+\alpha S^{\prime}(r)\right) w^{\prime}(r)+e^{-\alpha S(r)}\left(e^{-\alpha S(r)} w^{q}-\sigma\right)=0,
$$

and boundary conditions

$$
w^{\prime}(0)=0, w(R)=0,
$$


where we have set

$$
\sigma=2 \Omega^{2}
$$

We want to use the "time-map" method used by SMOLLER \& WASSERMAN(cf. [17]). For this purpose, we consider the problem (2.9) with initial data

$$
w(0)=p>0, w^{\prime}(0)=0
$$

where $p$ is a free parameter, and let $w(r, p, \sigma)$ be the solution of this problem. We define the " time-map" $p \rightarrow R(p, \sigma)$, by

$$
R(p, \sigma)=\inf \{R \mid R>0, w(r, p, \sigma)>0 \text { if } 0 \leq r<R \text {, and } w(R, p, \sigma)=0\},
$$

so $R(p, \sigma)$ is the first "time" at which $w$ is 0 (we will write $R(p, \sigma)=\infty$ if $w(r, p, \sigma)>0$ for all $r \geq 0)$. Thus $R(p, \sigma)$ is the radius of a rotating star with given central density (cf. (2.3))

$$
\rho(0)=\left(\frac{\gamma-1}{\gamma} p\right)^{\frac{1}{\gamma-1}} e^{-S(0) / \gamma} .
$$

and angular velocity

$$
\Omega=\sqrt{\sigma / 2}
$$

In order to state our first theorem, we introduce the following notation. We set

$$
\bar{S}=\sup _{r \geq 0} S(r), \underline{S}=\inf _{r \geq 0} S(r) .
$$

We assume

$$
-\infty<\underline{S} \leq \bar{S}<\infty
$$

We define the constant $b$ by

$$
b=\frac{(10+22 q) e^{-\frac{\alpha \bar{S}}{q}} \sigma^{\frac{q-1}{q}}}{\left(1+2 e^{\alpha \bar{S}}\right)[10(1+q)]^{\frac{1}{q}}(5-q)^{1-\frac{1}{q}}} .
$$

Obviously $b>0$ if $1<q<5$. Our first result is the following theorem.

Theorem 2.1. Assume that $D$ is a ball in $\mathbf{R}^{3}$ and that $\Omega$ is a nonzero constant.

(1) If $1<\gamma \leq 6 / 5$ and $S^{\prime}(r) \geq 0$ for $r \geq 0$, then

$$
w(r, p, \sigma)>0
$$

for every $r \geq 0$ and $p>0$. 
(2) If $4 / 3<\gamma<2$, then

$$
R(p, \sigma)<+\infty,
$$

for sufficiently large p, provided the entropy satisfies the following conditions:

$$
\alpha S^{\prime}(r) e^{\alpha S} z^{\prime}>-\frac{b}{2} z
$$

for $0<r<\pi / \sqrt{b}$, where

$$
z(r)=\frac{\sin (\sqrt{b} r)}{\sqrt{b} r},
$$

and $\sup _{0 \leq r<1+\frac{\pi}{\sqrt{b}}}\left|S^{\prime}(r)\right|$ is sufficiently small.

(3) If $6 / 5<\gamma<2$, assume that the conditions in (2) hold, and assume too that the entropy satisfies the following condition:

$$
S^{\prime}(r) \leq 0 \text { for } 0 \leq r \leq \frac{\pi}{\sqrt{b}}
$$

where $b$ is given by (2.16). Then

$$
R(p, \sigma)<+\infty
$$

for sufficiently large $p$.

(4) For the solutions $w(r, p, \sigma)=: w(r)$ in (2) and (3), we have the following estimates on the mass $M(r)=\int_{0}^{r} 4 \pi \tau^{2} \rho(\tau) d \tau$, and the average density $\bar{\rho}(r)=\frac{1}{\operatorname{Vol}_{r}(0)} M(r)$ in the ball $B_{r}(0)$ (here $\rho=\left[\frac{\gamma-1}{\gamma} w\right]^{1 /(\gamma-1)}$ is the density (cf. (2.3)) and Vol $\left.B_{r}(0)=4 \pi r^{3} / 3\right)$ :

$$
\begin{gathered}
M(r) \geq \frac{2 r^{3} \Omega^{2}}{3 G}, \\
\bar{\rho}(r) \geq \frac{\Omega^{2}}{2 \pi G},
\end{gathered}
$$

for $r \leq R(p, \sigma)$, where $G$ is the Newtonian gravitational constant.

Remark 1. The conditions imposed on the entropy $S(r)$ in Theorem 2.1 are automatically satisfied in the isentropic case, i.e., $S(r)=$ constant.

Remark 2. The existence of positive solutions for the equation of an isentropic non-rotating star is well known (cf. [5], [12] and [15]). In [8], for the case of a nonrotating $\operatorname{star}(\Omega=0)$, the authors first consider non-isentropic equations of state, and an existence theorem is proved for a bounded domain $D$, under the assumption that the entropy function $S$ satisfies, for some constant $a>0$,

$$
\int_{D}|\nabla \phi|^{2} e^{-S / \gamma} d x+\frac{1}{\gamma} \int_{D} \Delta S \phi^{2} e^{-S / \gamma} d x \geq a \int_{D} \phi^{2} e^{-S / \gamma} d x
$$

for all test functions $\phi \in H_{0}^{1}(D)$. Our condition (2.18) is an explicit condition on $S$ and does not involve the second derivative of $S$, for the case when domain $D$ is a ball. 
The proof this theorem, as well as the other theorems stated in this section will be given in Sections 3-5.

In the case of isentropic fluids (i.e., $S=$ constant), we can obtain further results on the qualitative properties of the solutions if the angular velocity $\Omega(\eta)=\Omega=$ constant and the domain $D$ is a ball. Without loss of generality, we may assume

$$
S=0 \text {. }
$$

In this case, it follows from the celebrated GIDAS, NI \& NIRENBERG result [9] that positive solutions to (2.5) and (2.7) must be spherically symmetric. Substituting $S=0$ in (2.9), we obtain the following equation:

$$
w^{\prime \prime}(r)+\frac{2}{r} w^{\prime}(r)+w^{q}-\sigma=0 .
$$

We consider the problem (2.25) with initial data

$$
w(0)=p>0, w^{\prime}(0)=0 .
$$

We will again use $w(r, p, \sigma)$ to denote the solution of the above problem. The following theorem gives some physically interesting properties of $R(p, \sigma)$, the radius of the star with central density (cf. (2.3) with $S=0$ )

$$
\rho(0)=\left(\frac{\gamma-1}{\gamma} p\right)^{\frac{1}{\gamma-1}}
$$

Theorem 2.2. Assume that $D$ is a ball in $\mathbf{R}^{3}, \Omega$ is a nonzero constant and the entropy $S$ is constant (set $S=0$ for convenience). Then the following statements hold:

(1) If $6 / 5<\gamma<2$, there exists a constant $p_{0}>0$ depending only on $\gamma$ and $\sigma$ such that

$$
R(p, \sigma)<+\infty \text { if and only if } p \geq p_{0} .
$$

Moreover, $p_{0}$ can be estimated from below:

$$
p_{0} \geq\left[\frac{5 \sigma(q+1)}{5-q}\right]^{1 / q}=\left[\frac{10 \gamma \Omega^{2}}{5 \gamma-6}\right]^{\gamma-1} .
$$

(2) If $6 / 5<\gamma<2$,

$$
R\left(p, \sigma_{1}\right) \geq R\left(p, \sigma_{2}\right),
$$

provided $\sigma_{1}>\sigma_{2}>0$, and

$$
R\left(p_{1}, \sigma\right) \geq R\left(p_{2}, \sigma\right)
$$

if $p_{2}>p_{1} \geq p_{0}$.

As a corollary of this result, we have the following theorem, in which $p_{0}$ is the positive constant given in Theorem 2.2. 
Theorem 2.3. Assume that $D$ is a ball in $\mathbf{R}^{3}, \Omega$ is a nonzero constant and the entropy $S$ is constant (set $S=0$ for convenience). If $6 / 5<\gamma<2$, then there exists a positive constant $C$ depending only on $p_{0}$ and $\Omega$ such that

$$
R(p, \sigma) \leq C p^{\frac{\gamma-2}{\gamma-1}} \leq C p_{0}^{\frac{\gamma-2}{\gamma-1}}
$$

for $p \geq p_{0}$. In particular, this implies

$$
R(p, \sigma) \rightarrow 0 \quad \text { as } p \rightarrow \infty
$$

We now discuss the physical meaning of the above two theorems.

\section{Physical meaning of Theorem 2.2}

Part (1) means, for $6 / 5<\gamma<2$ and $\sigma=2 \Omega^{2}>0$, there exits a critical central density, determined by $p_{0}$, depending only on $\gamma$ and the angular velocity $\Omega$, such that the radius of the star is finite if the central density is greater than or equal to this critical central density; otherwise the radius of the star is infinite. This is in sharp contrast to a non-rotating star, for which, in this range of $\gamma$, the radius is finite no matter how small the central density is (cf. [5]). The reason for this is that for the rotating star, the central density must be large enough to provide sufficient gravitational attraction to balance the centrifugal force due to rotation. The physical meaning of part (2) is that, for a compressible fluid, the radius of the star increases with increasing angular velocity for fixed central density, while the radius of the star decreases with increasing central density for the fixed angular velocity.

\section{Physical meaning of Theorem 2.3}

If $6 / 5<\gamma<2$, in order for the radius of the rotating star $R(p, \sigma)$ to be finite, $p$ has to be greater than or equal to $p_{0}$, and the constant $p_{0}$ is completely determined by $\gamma$ and the angular velocity $\Omega$, as stated in Theorem 1.2. Thus, (2.31) in Theorem 2.3 shows that there exists a (finite) upper bound on the radius of a rotating star, which is determined only by $\gamma$ and $\Omega$, and is independent of central density, for the radius of the rotating star to be finite. This is physically striking and completely different from the non-rotating star, for which the radius can be arbitrarily large. Actually, for the non-rotating star, the central density can also be arbitrarily small (cf. [5]). Moreover, as we will show later, the radius of a non-rotating star is proportional to $(\rho(0))^{\frac{\gamma-2}{2}}$, where $\rho(0)$ is the central density. Since $\gamma<2$, the radius of a non-rotating star can be very large if the central density is very small. However, for the rotating star, the central density cannot be arbitrarily small, as indicated in part (1) of Theorem 2.2; cf. (2.27). Moreover, (2.32) shows that the radius of a rotating star tends to zero if the central density tends to infinity.

We now turn to the case of general bounded domain $D \subset \mathbf{R}^{3}$ and variable angular velocity $\Omega(\eta)$. Without loss of generality, we may assume that $0 \in D$. For the existence of positive solutions to the boundary-value problem (2.5) and (2.7), we assume that the angular velocity $\Omega(\eta)$ is $C^{1}$ as a function of $\eta$ and satisfies the following condition: 
Condition A: Let $B_{R_{1}}(0)=\left\{x|| x \mid<R_{1}\right\}$ be the largest ball contained in $D$ which is centered at origin. For $x \in D-B_{R_{1}}(0)$, assume

$$
\Omega(\eta)[\eta \Omega(\eta)]^{\prime} \leq 0
$$

and is not identically zero; here, as before, $\eta=\eta(x)=\sqrt{x_{1}^{2}+x_{2}^{2}}$, and "prime" denotes $\frac{d}{d \eta}$.

Remark 3. Notice that (2.33) is only required for $x \in D-B_{R_{1}}(0)$. Therefore the angular velocity $\Omega(\eta)$ can be any $C^{1}$ function when $D$ is a ball.

The following theorem is an existence theorem for a general domain $D$ and variable angular velocity.

Theorem 2.4. Assume $6 / 5<\gamma<2$, the entropy $S$ is constant (set $S=0$ for convenience), $\Omega(\eta(x)) \in C^{1+\delta}(D) \cap C(\bar{D})$ for some $\delta, 0<\delta<1$ with $\partial D$ smooth. Then there exists a constant $R_{2}>0$ depending only on $\Omega$ and $\gamma$ such that if $D \subset B_{R_{2}}(0)$ and condition A holds, there exists a positive solution to (2.5) and (2.7). Moreover, for this solution, the central density satisfies

$$
\rho(0) \geq\left(\frac{\gamma-1}{\gamma}\right)^{1 /(\gamma-1)} \cdot \frac{5 \gamma}{5 \gamma-6} \cdot \beta,
$$

where $\beta=\max _{x \in \bar{D}} \mid 2 \Omega(\eta)\left(\Omega(\eta)+\eta \Omega^{\prime}(\eta) \mid\right.$.

Notice that as in Theorem 2.3 , the size of the domain $D$ is uniformly bounded, where the bound depends only on $\gamma$ and $\Omega$.

Let $w(x)$ be the positive solution given in Theorem 2.4. Notice that since $S=0$, (2.3) implies $\rho=\left(\frac{\gamma-1}{\gamma} w\right)^{\frac{1}{\gamma-1}}$. We can obtain the potential function by virtue of (1.10) and (1.13). Actually, when $S=0,(1.10)$ and (1.13) imply $\nabla(w+\Phi-J)=0$. So we set $\Phi(x)=(C+w+J(\eta))(x)$ for $x \in D$, where $C$ is any constant. Then $(\rho, \Phi)(x)$ is a positive solution to the system (1.3) in the domain $D$. System (1.3) is derived by assuming that $\rho$ is axi-symmetric. In fact, we have the following self consistency result.

Theorem 2.5. Suppose the conditions in Theorem 2.4 hold. If the domain $D$ is axi-symmetric about the $x_{3}$-axis, then the solution $w(x)$ in Theorem 2.4 is also axi-symmetric about the $x_{3}$-axis.

Our final result is the following non-existence theorem; here $v(x)$ denotes the unit outer normal vector on the boundary, and $\eta=\eta(x)=\sqrt{x_{1}^{2}+x_{2}^{2}}$;

Theorem 2.6. Suppose $\partial D \in C^{1}$ and the domain $D$ is bounded and star-shaped, i.e., $x \cdot v(x) \geq 0$ for $x \in \partial D$, and the entropy $S$ satisfies the condition

$$
x \cdot \nabla S(x) \geq 0 \quad \text { for } x \in D .
$$

If $\gamma \leq 6 / 5($ or $\gamma<6 / 5)$ and

$$
\eta A^{\prime}(\eta)+\frac{5 A(\eta)}{2}>0(\text { resp. } \geq 0)
$$

for $x \in D$, where $A(\eta)=2 \Omega(\eta)(\eta \Omega(\eta))^{\prime}$, then there is no positive solution to (2.5) and (2.7). 
Remark 4. Condition (2.36) is trivially satisfied when $\Omega$ is a constant.

Remark 5. In [8], for the case of a non-rotating star $(\Omega=0)$, the authors prove a non-existence theorem for $1<\gamma<6 / 5$, under the assumption that the entropy $S(x)$ satisfies the following three conditions, for all $x \in D$ :

$$
x \cdot \nabla S \geq 0, \Delta S \geq 0 \text { and } \nabla(\Delta S) \cdot x \geq 0 .
$$

In Theorem 2.6, we only require $x \cdot \nabla S \geq 0$ for all $x \in D$.

We now make some remarks about the above theorems and their proofs. In the theory of second-order elliptic equations, the existence of a positive solution to the boundary-value problem

$$
\begin{aligned}
\Delta w+f(x, w) & =0, x \in D, \\
\left.w\right|_{\partial D} & =0,
\end{aligned}
$$

where $D \in R^{n}$ is a bounded open set, has been extensively studied either by the Min-Max method of the Mountain Pass Lemma of RABINOwITZ [15] or the Topological Degree Method (Leray-Schauder degree) by LIONS [12], both under the condition $f(x, 0) \geq 0$. If $f(x, 0)<0$, the difficulty in solving the boundary-value problem (2.37) is that the Harnack inequality is not applicable, and thus the above variational methods (Mountain Pass Lemma) and the Topological Degree Method do not work in this case. The first existence result of a positive solution for the case $f(x, 0)<0$ was given in [17] by using the time-map method when $D$ is a ball and $f(x, w)=f(w)$ (i.e., $f$ does not depend on $x$ explicitly); this corresponds to the case when both the entropy $S$ and the angular velocity $\Omega$ are constant in equation (2.5). For this type of equation, further analysis was given in [4]. The existence result of a spherically symmetric solution to (2.5) and (2.8) generalizes the above-mentioned results to the non-isentropic case.

If $D$ is a general domain, [17] contains some existence results for the case $f(x, w)=f(w)$ and $f$ is sublinear in $w$. To the best of our knowledge, Theorem 2.4 is the first result of existence of positive solutions for the case where $f(x, w)$ is superlinear in $w$ and the domain $D$ is different from a ball.

The rest of this paper is organized as follows. In Section 3, we prove Theorem 2.1. Theorems 2.2 and 2.3 are proved in Section 4. Theorems 2.4-2.6 are proved in Section 5. Further discussions are given in Section 6.

\section{Proof of Theorem 2.1}

In this section, we shall prove Theorem 2.1. We define

$$
f(r, w)=\left\{\begin{array}{cl}
e^{-\alpha S(r)} w^{q}-\sigma & \text { if } w \geq 0 \\
-\sigma & \text { if } w<0
\end{array}\right.
$$

and

$$
F(r, w)=\int_{0}^{w} f(r, z) d z
$$


As in Section 2, we use the notation $w(r, p, \sigma)$ to denote the solution of the problem

$$
w^{\prime \prime}(r)+\left(\frac{2}{r}+\alpha S^{\prime}(r)\right) w^{\prime}(r)+e^{-\alpha S} f(r, w)=0
$$

and

$$
w^{\prime}(0)=0, w(0)=p>0 .
$$

Let $R(p, \sigma)$ be as in (2.13); i.e., $R(p, \sigma)$ the first point at which $w$ is 0 .

Motivated by the celebrated POHOZAEV identity [14], we define the function $G(r)$ by

$$
\begin{aligned}
G(r)= & r^{3}\left(e^{\alpha S(r)} \frac{w^{\prime 2}}{2}+F(r, w)\right)+\frac{1}{2} r^{2} e^{\alpha S} w w^{\prime}(r) \\
& +\frac{\alpha}{2} \int_{0}^{r} t^{3} e^{\alpha S} S^{\prime} w^{\prime 2} d t, r \geq 0 .
\end{aligned}
$$

It is easy to verify that

$$
G^{\prime}(r)=3 r^{2}\left(F(r, w)-\frac{f(r, w) w}{6}\right)+r^{3} g(r, w),
$$

where

$$
g(r, w)=\int_{0}^{w} \frac{\partial f(r, z)}{\partial r} d z=\left\{\begin{array}{cl}
-\frac{\alpha S^{\prime}(r) e^{-\alpha S} w^{q+1}}{q+1}, & w \geq 0 \\
0, & w<0
\end{array}\right.
$$

thus

$$
G(r)=\int_{0}^{r}\left\{3 t^{2}\left[F(t, w)-\frac{f(t, w) w}{6}\right]+t^{3} g(t, w)\right\} d t, r \geq 0 .
$$

We now prove that

$$
\begin{gathered}
\text { If } 1<\gamma \leq 6 / 5(q \geq 5) \text { and } S^{\prime}(r) \geq 0 \text { for } r \geq 0, \text { then } \\
R(p, \sigma)=+\infty
\end{gathered}
$$

for any $p>0$ and $\sigma>0$.

The proof is by contradiction. Suppose $R(p, \sigma)<+\infty$, and let $\bar{R}=R(p, \sigma))$. Thus

$$
w(\bar{R})=0, w(r)>0 \text { for } 0 \leq r<\bar{R} .
$$

By the definition of $G(r)(2.5)$, we have

$$
G(\bar{R})=\frac{\bar{R}^{3} e^{\alpha S(\bar{R})}\left(w^{\prime}(\bar{R})\right)^{2}}{2}+\frac{\alpha}{2} \int_{0}^{\bar{R}} r^{3} e^{\alpha S} S^{\prime}(r) w^{\prime 2} d r .
$$


Applying this to (2.6), we obtain, by virtue of (3.1) and (3.2),

$$
\begin{aligned}
& \frac{\bar{R}^{3} e^{\alpha S(\bar{R})}\left(w^{\prime}(\bar{R})\right)^{2}}{2}+\frac{\alpha}{2} \int_{0}^{\bar{R}} r^{3} e^{\alpha S} S^{\prime}(r) w^{\prime 2} d r \\
& =\int_{0}^{\bar{R}} 3 r^{2}\left\{\left[\left(\frac{1}{q+1}-\frac{1}{6}\right) e^{-\alpha S} w^{q+1}-\frac{5}{6} \sigma w\right]-r^{3} \frac{\alpha}{q+1} S^{\prime}(r) e^{-\alpha S} w^{q+1}\right\} d r .
\end{aligned}
$$

Since $\frac{1}{q+1}-\frac{1}{6} \leq 0$ for $q \geq 5$ and $S^{\prime}(r) \geq 0$, (3.11) gives a contradiction. Thus (3.9) holds so part (1) in Theorem 2.1 is proved.

Next, we give the proof of parts (2) and (3) of Theorem 2.1. First, it follows from (3.3) that

$$
\left(r^{2} e^{\alpha S} w^{\prime}\right)^{\prime}+r^{2} f(r, w)=0
$$

Thus from (3.12) and (2.4), we obtain

$$
w^{\prime}(r)=-\frac{e^{-\alpha S(r)}}{r^{2}} \int_{0}^{r} t^{2} f(t, w(t)) d t, r \geq 0 .
$$

For fixed $p>0$, since $w(0)=p$, by (3.1), (3.2) and (3.13), we have $w^{\prime}(r)<0$ for small $r, r>0$, if $p$ is sufficiently large. We define $r_{1}$ to be the point such that

$$
w\left(r_{1}\right)=2 p / 3, w(r)>2 p / 3 \text { for } 0<r<r_{1} .
$$

Then we have the following lemma which estimates $r_{1}$ and $G\left(r_{1}\right)$ in terms of $p$.

Lemma 3.1. For sufficiently large $p$, if $1<q<5(6 / 5<\gamma<2)$, then there are positive constants $c_{1}, c_{2}$ and $c_{3}$ independent of $p$ such that

$$
c_{1} p^{-(q-1) / 2} \leq r_{1} \leq c_{2} p^{-(q-1) / 2},
$$

and

$$
G\left(r_{1}\right) \geq c_{3} p^{(5-q) / 2},
$$

provided $\sup _{0 \leq r \leq r_{1}}\left|S^{\prime}(r)\right|$ is sufficiently small.

Proof. By the definition of $r_{1}$ (cf. (3.14)), we have

$$
2 p / 3 \leq w(r) \leq p, \quad 0 \leq r \leq r_{1} .
$$

Thus, from (3.1), (3.13) and (3.17),

$$
\begin{aligned}
2 p / 3 & =w\left(r_{1}\right)=p-\int_{0}^{r_{1}} \frac{e^{-\alpha S(r)}}{r^{2}} \int_{0}^{r} t^{2}\left(e^{-\alpha S(t)} w^{q}(t)-\sigma\right) d t d r \\
& \geq p-\int_{0}^{r_{1}} \frac{e^{-\alpha \underline{S}}}{r^{2}} \int_{0}^{r} t^{2}\left(e^{-\alpha \underline{S}} p^{q}-\sigma\right) d t d r \\
& =p-\frac{r_{1}^{2}}{6}\left(e^{-2 \alpha \underline{S}} p^{q}-e^{-\alpha \underline{S}} \sigma\right)
\end{aligned}
$$


if $p$ is sufficiently large, where $\underline{S}=\inf _{0 \leq r<+\infty} S(r)$, and by (2.15) $\underline{S}>-\infty$. From (3.18), we get

$$
\frac{r_{1}^{2}}{2}\left(e^{-2 \alpha \underline{S}} p^{q}-e^{-\alpha \underline{S}} \sigma\right) \geq p
$$

This implies

$$
r_{1} \geq c_{1} p^{-(q-1) / 2}
$$

for some positive constant $c_{1}$, if $p$ is sufficiently large. Similar to the argument in (3.18), we can obtain,

$$
2 p / 3 \leq p-\frac{r_{1}^{2}}{6}\left(e^{-2 \alpha \bar{S}}(2 p / 3)^{q}-e^{-\alpha \bar{S}} \sigma\right)
$$

where $\bar{S}=\sup _{0 \leq r<+\infty} S(r)$, and $\bar{S}<\infty$. So from (3.20),

$$
r_{1} \leq c_{2} p^{-(q-1) / 2}
$$

for some positive constant $c_{2}$, if $p$ is sufficiently large. This proves (3.15). To prove (3.16), we have, in view of (3.8),

$$
G\left(r_{1}\right)=\int_{0}^{r_{1}} 3 r^{2}\left\{\left[\frac{1}{q+1}-\frac{1}{6}-\frac{\alpha}{3(q+1)} r S^{\prime}(r)\right] e^{-\alpha S} w^{q+1}-\frac{5}{6} \sigma w\right\} d r .
$$

Since $1<q<5(q+1<6)$, we have

$$
\frac{1}{q+1}-\frac{1}{6}-\frac{\alpha}{3(q+1)} r S^{\prime}(r)>c_{3}, \quad 0 \leq r \leq r_{1},
$$

if $\sup _{0 \leq r<r_{1}}\left|S^{\prime}(r)\right|$ is sufficiently small, where $c_{3}$ is some positive constant independent of $p$. By virtue of (3.14), (3.21) and (3.22), we have,

$$
\begin{aligned}
G\left(r_{1}\right) & \geq r_{1}^{3}\left(c_{3} e^{-\alpha \bar{S}}\left(w\left(r_{1}\right)\right)^{q+1}-\frac{5}{6} \sigma p\right) \\
& =r_{1}^{3}\left(c_{3} e^{-\alpha \bar{S}}\left((2 p / 3)^{q+1}-\frac{5}{6} \sigma p\right) .\right.
\end{aligned}
$$

Therefore, if $p$ is sufficiently large, (3.23) implies (3.16). This proves Lemma 3.2.

By (3.1), we have

$$
f(r, w)>0 \text { for } w>\left[e^{\alpha \bar{S}} \sigma\right]^{1 / q} .
$$

Therefore, in view of (3.13), we have $w^{\prime}(r)<0$ for small $r$ if $p$ is sufficiently large, so $w(r)$ decreases for small $r$. Using (3.13) and (3.24), we can see that $w^{\prime}(r)<0$ 
and thus $w(r)$ decreases as long as $w>\left[e^{\alpha \bar{S}} \sigma\right]^{1 / q}$. Because of this, we can define $T=: T(p)$ as the point such that

$$
w(T)=\left[\frac{10(q+1) e^{\alpha \bar{S}} \sigma}{(5-q)}\right]^{1 / q}=: A, w(r)>A \text { for } 0 \leq r<T,
$$

since

$$
w(T)=A>\left[e^{\alpha \bar{S}} \sigma\right]^{1 / q},
$$

because $1<q<5$. So by (3.1) and (3.25), we have

$$
f(r, w(r))>0,0 \leq r \leq T,
$$

and thus

$$
w^{\prime}(r)<0 \quad \text { for } 0<r \leq T .
$$

Remark 6. The existence of such a $T$ follows here by an argument similar to that in [17].

The next lemma gives an upper bound for $T$, where the constant $b$ is given by (2.16).

Lemma 3.2. Assume that $1<q<5(6 / 5<\gamma<2)$ and

$$
\alpha S^{\prime}(r) e^{\alpha S} z^{\prime}>-\frac{b}{2} z
$$

for $0<r<\pi / \sqrt{b}$, where

$$
z(r)=\frac{\sin (\sqrt{b} r)}{\sqrt{b} r}
$$

Then

$$
T \leq \pi / \sqrt{b}
$$

Proof. It is easy to verify that $z(r)$ defined in (3.29) is the solution of the following initial-value problem of second-order linear equation:

$$
z^{\prime \prime}+\frac{2}{r} z^{\prime}+b z=0, \quad z(0)=1, z^{\prime}(0)=0,
$$

By (3.12) and (3.31), we have

$$
\left[r^{2} e^{\alpha S(r)}\left(z^{\prime} w-w^{\prime} z\right)\right]^{\prime}=r^{2}\left[f(r, w) z-b z e^{\alpha S} w+\alpha S^{\prime}(r) e^{\alpha S} z^{\prime} w\right]
$$

We prove (3.30) by contradiction. Suppose $T>\pi / \sqrt{b}$, then in view of (3.1), (3.26) and (3.27), we have

$$
\frac{f(r, w(r))}{w(r)}-b e^{\alpha S(r)} \geq e^{-\alpha \bar{S}}(w(T))^{q-1}-\frac{\sigma}{w(T)}-b e^{\alpha \bar{S}}=\frac{b}{2} .
$$


Integrating (3.32) over the interval $[0, \pi / \sqrt{b}]$, noticing the fact $z(\pi / \sqrt{b})=0$, we obtain

$$
\left.r^{2} e^{\alpha S(r)} z^{\prime} w\right|_{r=\pi / \sqrt{b}}=\int_{0}^{\pi / \sqrt{b}} r^{2} w z\left\{\frac{f(r, w)}{w}-b e^{\alpha S}+\frac{\alpha S^{\prime}(r) e^{\alpha S} z^{\prime}}{z}\right\} d r .
$$

In view of (3.27), we have $w\left(\frac{\pi}{\sqrt{b}}\right)>w(T)>0$ if $T>\frac{\pi}{\sqrt{b}}$. Moreover from (3.29) $z^{\prime}\left(\frac{\pi}{\sqrt{b}}\right)<0$. Thus the left-hand side of (3.34) is negative. On the other hand, by (3.28) and (3.33), we can see that the right-hand side of (3.34) is positive. This is a contradiction, and thus (3.30) is proved.

In order to prove parts (2) and (3) in Theorem 2.1, we need a few lemmas. First, in view of (3.1) and (3.2), there exists a positive constant $B$ such that

$$
\begin{array}{r}
\left|e^{-\alpha S(r)} F(r, w)\right| \leq B \text { for } 0 \leq w \leq A, \\
e^{-\alpha S(r)}\left|g(r, w)-\alpha S^{\prime}(r) F(r, w)\right| \leq B \text { for } 0 \leq w \leq A,
\end{array}
$$

where $A=w(T)$ is defined in (3.25), $g(r, w)$ is defined in (3.7). In the following, we denote

$$
Q:=Q(T)=w^{\prime}(T)
$$

where $T=T(p)$ is defined in (3.25). By (3.27), we have

$$
Q<0
$$

Lemma 3.3. If the entropy $S$ satisfies the hypothesis in Lemma 2.2, and

$$
\frac{4 B}{Q^{2}}\left(1+\frac{A}{|Q|}\right)+\frac{12 A}{|Q| T}\left(1+\frac{4 B}{Q^{2}}+\frac{4 A B}{|Q|^{3}}\right)<\frac{1}{2},
$$

and

$$
0 \leq w(r) \leq A=w(T) \text { for } T \leq r \leq T+L,
$$

for any L satisfying

$$
0 \leq L \leq \min \left\{\frac{2 A}{|Q|}, 1\right\}
$$

then

$$
w^{\prime}(r) \leq-\frac{|Q|}{\sqrt{2}} \text { for } T \leq r \leq T+L
$$

provided $\sup _{0 \leq r<1+\frac{\pi}{\sqrt{b}}}\left|S^{\prime}(r)\right|$ is sufficiently small. 
Proof. First, by (3.3), we have

$$
\begin{aligned}
& \left(\frac{w^{\prime 2}}{2}+e^{-\alpha S} F(r, w)\right)^{\prime} \\
& =-\frac{2+\alpha r S^{\prime}(r)}{r} w^{\prime 2}+e^{-\alpha S(r)}\left[g(r, w)-\alpha S^{\prime}(r) F(r, w)\right]
\end{aligned}
$$

where $g(r, w)$ is given by (3.7). Notice that $T$ is bounded by $\pi / \sqrt{b}$ (cf. (2.30)), where $b$ is given by (2.16), so

$$
[T, T+1] \subset\left[0, \frac{\pi}{\sqrt{b}}+1\right] .
$$

This, together with (3.41), implies

$$
[T, T+L] \subset\left[0, \frac{\pi}{\sqrt{b}}+1\right] .
$$

Therefore, if $\sup _{0 \leq r<1+\frac{\pi}{\sqrt{b}}}\left|S^{\prime}(r)\right|$ is sufficiently small, then $\left|r S^{\prime}(r)\right|$ is also small for $T \leq r \leq T+L$. Thus

$$
0<2+\alpha r S^{\prime}(r)<3
$$

for $T \leq r \leq T+L$. It follows from (3.43) and (3.46) that

$$
\begin{aligned}
\frac{w^{\prime 2}}{2}+e^{-\alpha S} F(r, w) \leq & \frac{Q^{2}}{2}+e^{-\alpha S(T)} F(T, w(T)) \\
& +\int_{T}^{r}\left[e^{-\alpha S(t)}\left(g(t, w)-\alpha S^{\prime}(t) F(t, w)\right] d t\right.
\end{aligned}
$$

for $T \leq r \leq T+L$. Using (3.35), (3.36) and (3.47), we obtain

$$
\frac{w^{\prime 2}}{2} \leq \frac{Q^{2}}{2}+2 B+B L \leq \frac{Q^{2}}{2}+2 B+\frac{2 B A}{|Q|}
$$

for $T \leq r \leq T+L$. On the other hand, we have, from (3.36) and (3.43),

$$
\begin{aligned}
\frac{w^{\prime 2}}{2}+e^{-\alpha S(r)} F(r, w) \geq & \frac{Q^{2}}{2}+e^{-\alpha S(T)} F(T, w(T)) \\
& -\int_{T}^{r} \frac{2+\alpha t S^{\prime}(t)}{t}\left(w^{\prime}(t)^{2} d t-B L\right.
\end{aligned}
$$

for $T \leq r \leq T+L$. By (3.35), (3.46) and (3.49), we obtain

$$
\begin{aligned}
\frac{w^{\prime 2}}{2} & \geq \frac{Q^{2}}{2}-2 B-B L-\int_{T}^{T+L} \frac{3}{t}\left(w^{\prime}(t)^{2} d t\right. \\
& \geq \frac{Q^{2}}{2}-2 B\left(1+\frac{L}{2}\right)-\int_{T}^{T+L} \frac{3}{T}\left(w^{\prime}(t)\right)^{2} d t
\end{aligned}
$$


for $T \leq r \leq T+L$. So, if $L$ satisfies (3.41), by virtue of (3.48), we have

$$
\begin{aligned}
\frac{w^{\prime 2}}{2} & \geq \frac{Q^{2}}{2}-2 B\left(1+\frac{A}{|Q|}\right)-\int_{T}^{T+\frac{2 A}{|Q|}} \frac{3}{T}\left(w^{\prime}(t)^{2} d t \quad(\text { by }(3.41))\right. \\
& \geq \frac{Q^{2}}{2}-2 B\left(1+\frac{A}{|Q|}\right)-\int_{T}^{T+\frac{2 A}{|Q|}} \frac{3}{T}\left(Q^{2}+4 B+\frac{4 B A}{|Q|}\right) d t \quad(\text { by (3.48)) } \\
& =\frac{Q^{2}}{2}-2 B\left(1+\frac{A}{|Q|}\right)-\frac{6 A}{T|Q|}\left(Q^{2}+4 B+\frac{4 B A}{|Q|}\right)
\end{aligned}
$$

for $T \leq r \leq T+L$. Hence

$$
\frac{w^{\prime 2}}{Q^{2}} \geq 1-\frac{4 B}{Q^{2}}\left(1+\frac{A}{|Q|}\right)-\frac{12 A}{T|Q|}\left(1+\frac{4 B}{Q^{2}}+\frac{4 B A}{|Q|^{3}}\right)
$$

for $T \leq r \leq T+L$. Therefore, if (3.39) holds, then we have

$$
\left(w^{\prime}(r)\right)^{2} \geq \frac{Q^{2}}{2}
$$

for $T \leq r \leq T+L$. This implies $w^{\prime}(r)$ does not change sign for $T \leq r \leq T+L$. Since $Q=w^{\prime}(T)<0$ (cf. (3.27)),

$$
w^{\prime}(r) \leq-\frac{|Q|}{\sqrt{2}}
$$

for $T \leq r \leq T+L$

Next, we have the following lemma.

Lemma 3.4. If $w(r)>0$ for $r \in[T, T+L]$ where L satisfies (3.41), then

$$
0<w(r)<A \text { for } T<r \leq L
$$

Proof. Since $w(T)=A$ and $w^{\prime}(T)=Q<0$ as we showed before, then $w^{\prime}(r)<$ 0 , and thus $w(r)<A$ for $r>T,(r-T)$ small. We prove (3.55) by contradiction. If (3.55) were false, then there would exist $r_{2} \in(T, T+L]$ such that

$$
w(r)<A \text { for } r \in\left(T, r_{2}\right), w\left(r_{2}\right)=A .
$$

Since $w(T)=w\left(r_{2}\right)=A$ and $r_{2}>T$, by Rolle's Theorem, we have

$$
w^{\prime}(\tau)=0
$$

for some $\tau \in\left(T, r_{2}\right)$. This contradicts (3.54). The proof of the lemma is complete. 
The following lemma is a generalization of a result in [17].

Lemma 3.5. Assume the entropy $S$ satisfies the hypothesis in Lemma 3.2 . Let $T(p)=T$ be the point defined in (3.25). If

$$
w^{\prime}(T) T \rightarrow-\infty \text { as } p \rightarrow+\infty,
$$

then $R(p, \sigma)<+\infty$ if $p$ is sufficiently large and $\sup _{0 \leq r \leq 1+\frac{\pi}{\sqrt{b}}}\left|S^{\prime}(r)\right|$ is sufficiently small.

Proof. First, in view of (3.30), (3.58) implies

$$
Q(T)=w^{\prime}(T) \rightarrow-\infty \text { as } p \rightarrow+\infty .
$$

Hence, there exists $p_{0}>0$ such that (3.39) holds for every $p \gtrsim p_{0}$. Now, for any fixed $p \in\left[p_{0},+\infty\right)$, we show that there exists $r^{*} \in\left[T, T+\frac{\overline{2 A}}{|Q|}\right]$ such that

$$
w\left(r^{*}\right) \leq 0,
$$

and this implies $R(p, \sigma)<+\infty$. We prove (3.60) by contradiction. Suppose

$$
w(r)>0 \text { for } r \in\left[T, T+\frac{2 A}{|Q|}\right] .
$$

Then by Lemma 3.4, we have

$$
0<w(r)<A \text { for } r \in\left[T, T+\frac{2 A}{|Q|}\right] .
$$

Thus, we can apply Lemma 3.3 with $L=T+\frac{2 A}{|Q|}$ to obtain

$$
w^{\prime}(r) \leq-\frac{|Q|}{\sqrt{2}} \text { for } T \leq r \leq T+\frac{2 A}{|Q|} .
$$

Therefore, since $w(T)=A$, we have

$$
w\left(T+\frac{2 A}{|Q|}\right)=A+\int_{T}^{T+\frac{2 A}{|Q|}} w^{\prime}(r) d r \leq A-\frac{|Q|}{\sqrt{2}} \cdot \frac{2 A}{|Q|}<0 .
$$

This contradicts (3.61). The proof of the lemma is complete.

For the case $1<q<3(4 / 3<\gamma<2)$, we have the following result. This is the same as part (2) in Theorem 2.1.

Proposition 3.6. Assume $1<q<3(4 / 3<\gamma<2)$ and $\sigma>0$. If the entropy $S(r)$ satisfies condition (3.28) in Lemma 3.2, then

$$
R(p, \sigma)<+\infty
$$

for sufficiently large $p$, provided $\sup _{0 \leq r<1+\frac{\pi}{\sqrt{b}}}\left|S^{\prime}(r)\right|$ is sufficiently small. 
Proof. To prove this proposition, it suffices to verify (3.58) in Lemma 3.5. This follows by the following argument. Set

$$
w^{\prime}(T)=Q
$$

By (3.13), we have

$$
-e^{\alpha S(T)} T^{2} Q=\int_{0}^{T} r^{2} f(r, w(r)) d r .
$$

We estimate $Q T^{2}$ as follows. For $0 \leq r \leq T$, by (3.27), we have

$$
w(r) \geq w(T) .
$$

Therefore, by (3.1) and (3.25), we obtain

$$
\begin{aligned}
f(r, w(r)) & =e^{-\alpha S(r)} w^{q}(r)-\sigma \\
& \geq e^{-\alpha \bar{S}} w^{q}(r)-\sigma \\
& \geq e^{-\alpha \bar{S}} w^{q}(T)-\sigma \\
& \geq \frac{(5+11 q) \sigma}{5-q}>0
\end{aligned}
$$

for $0 \leq r \leq T$, since $1<q<3$. On the other hand, for $r_{1}$ defined in (3.14), since $2 p / 3>A=w(T)$ if $p$ is sufficiently large, then (3.25) and (3.27) imply

$$
T>r_{1}
$$

for $p$ large. Hence, it follows from (3.66)-(3.69) that

$$
-e^{\alpha S(T)} T^{2} Q \geq \int_{0}^{r_{1}} r^{2} f(r, w(r)) d r .
$$

By (3.14), we have

$$
w(r) \geq w\left(r_{1}\right) \geq 2 p / 3 \quad \text { for } 0 \leq r \leq r_{1} .
$$

Thus, by (3.1),

$$
f(r, w(r)) \geq e^{\alpha \bar{S}}(3 p / 2)^{q}-\sigma \geq \mathrm{const} \cdot p^{q} \quad \text { for } 0 \leq r \leq r_{1},
$$

if $p$ is sufficiently large. Therefore, we obtain, by (3.15), (3.70) and (3.72),

$$
-e^{\alpha S(T)} T^{2} Q \geq \mathrm{const} \cdot p^{q} r_{1}^{3}=\geq \mathrm{const} \cdot p^{\frac{3-q}{2}} .
$$

This implies $T^{2} Q \rightarrow-\infty$ as $p \rightarrow+\infty$ if $q<3$. Condition (3.58) is thus verified in view of (3.30) in Lemma 3.2.

For the case where $1<q<5(6 / 5<\gamma<2)$, we have the following result. This is the same as part (3) in Theorem 2.1. 
Proposition 3.7. Assume $1<q<5(6 / 5<\gamma<2)$ and $\sigma>0$. If the entropy $S(r)$ satisfies the hypothesis in Proposition 3.6 and

$$
S^{\prime}(r) \leq 0 \text { for } 0 \leq r \leq \frac{\pi}{\sqrt{b}}
$$

where $b$ is given by (2.16), then

$$
R(p, \sigma)<+\infty
$$

for sufficiently large $p$, provided $\sup _{0 \leq r \leq 1+\frac{\pi}{\sqrt{b}}}\left|S^{\prime}(r)\right|$ is sufficiently small.

Proof. First, in view of (3.14) and (3.25), we have

$$
w\left(r_{1}\right)>w(T)
$$

if $p$ is sufficiently large. This, together with (3.27), implies

$$
r_{1}>T
$$

Once again, by (3.25), we obtain

$$
w(r) \geq w(T)
$$

for $r_{1} \leq r \leq T$. It follows from (3.6) that

$$
G(T)=G\left(r_{1}\right)+\int_{r_{1}}^{T} 3 r^{2}\left(F(r, w)-\frac{1}{6} f(r, w) w+\frac{1}{3} r g(r, w)\right) d r .
$$

By (3.1), (3.2), (3.7), we have

$$
\begin{aligned}
& F(r, w)-\frac{1}{6} f(r, w) w+\frac{1}{3} r g(r, w) \\
& \quad=\left\{\left[\frac{1}{q+1}-\frac{1}{6}-\frac{\alpha r S^{\prime}(r)}{3(q+1)}\right] e^{-\alpha S}(w(r))^{q}-\frac{5}{6} \sigma\right\} w(r)
\end{aligned}
$$

for $r_{1} \leq r \leq T$. If $\sup _{0 \leq r \leq 1+\frac{\pi}{\sqrt{b}}}\left|S^{\prime}(r)\right|$ is small, in view of (3.30), we have

$$
\frac{1}{q+1}-\frac{1}{6}-\frac{\alpha r S^{\prime}(r)}{3(q+1)}>\frac{1}{2}\left(\frac{1}{q+1}-\frac{1}{6}\right)>0
$$

for $r \leq T$ and $1<q<5$. Moreover, by (3.25), (3.79), (3.78) and (3.81), we obtain

$$
\begin{aligned}
& F(r, w)-\frac{1}{6} f(r, w) w+\frac{1}{3} r g(r, w) \\
& \quad=\left\{\frac{1}{2}\left[\frac{1}{q+1}-\frac{1}{6}\right] e^{-\alpha S}(w(T))^{q}-\frac{5}{6} \sigma\right\} w(r) \geq 0
\end{aligned}
$$

for $r_{1} \leq r \leq T$. This, together with (3.16) and (3.79), implies

$$
G(T) \geq G\left(r_{1}\right) \geq c_{3} p^{(5-q) / 2} .
$$


By (2.5) and (3.83), we have

$$
\begin{gathered}
\frac{e^{\alpha S(T)} T}{2}\left([T Q]^{2}+(T)^{3} F(T, w(T))+\frac{T A e^{\alpha S(T)}}{2}[T Q]\right. \\
+\frac{\alpha}{2} \int_{0}^{T} r^{3} e^{\alpha S(r)} S^{\prime}(r)\left(w^{\prime}(r)\right)^{2} d r \geq c_{3} p^{(5-q) / 2},
\end{gathered}
$$

where $Q=w^{\prime}(T)$. So, if $S^{\prime}(r) \leq 0$ for $0 \leq r \leq \frac{\pi}{\sqrt{b}}$, we have, in view of (3.30),

$$
\frac{e^{\alpha S(T)} T}{2}[T Q]^{2}+(T)^{3} F(T, w(T))+\frac{T A e^{\alpha S(T)}}{2}[T Q] \geq c_{3} p^{(5-q) / 2},
$$

Since $T \leq \frac{\pi}{\sqrt{b}}$ and $1<q<5$, we have

$$
T Q \rightarrow-\infty \text {, as } p \rightarrow+\infty \text {. }
$$

By virtue of Lemma 3.5, Proposition 3.7 is proved.

Now we prove part (4) of Theorem 2.1. For the solutions $w(r, p, \sigma)=: w(r)$ in (2) and (3) of Theorem 2.1, let $\rho=\left[\frac{\gamma-1}{\gamma} w\right]^{1 /(\gamma-1)} ; \rho$ is the density function (cf. (2.3)). Set

$$
M(r)=\int_{0}^{r} 4 \pi \tau^{2} \rho(\tau) d \tau,
$$

the mass in the ball $B_{r}(0)$. We calculate each term in (2.5) as follows, by virtue of the fact that $w$ and $S$ are spherically symmetric and $\Omega$ is a constant. First, for $r=\sqrt{x_{1}^{2}+x_{2}^{2}+x_{3}^{2}}$, we have

$$
\nabla w=w_{r}\left(x_{1} / r, x_{2} / r, x_{3} / r\right), \quad \nabla S=S_{r}\left(x_{1} / r, x_{2} / r, x_{3} / r\right),
$$

and thus

$$
\begin{aligned}
\operatorname{div}\left(e^{\alpha S} \nabla w\right) & =\nabla\left(e^{\alpha S}\right) \cdot \nabla w+e^{\alpha S} \Delta w \\
& =\alpha e^{\alpha S} w_{r} S_{r}+e^{\alpha S}\left(w_{r r}+\frac{2}{r} w_{r}\right) \\
& =e^{\alpha S} w_{r r}+e^{\alpha S}\left(\frac{2}{r}+\alpha S^{\prime}(r)\right) w_{r} .
\end{aligned}
$$

By (2.3) and (2.6), we have

$$
K e^{-\alpha S} w^{q}=4 \pi G \rho .
$$

Substituting (3.88) and (3.89) into (2.5), and noticing $\Omega$ is a constant, we obtain

$$
e^{\alpha S} w_{r r}+e^{\alpha S}\left(\frac{2}{r}+\alpha S^{\prime}(r)\right) w_{r}+4 \pi G \rho-2 \Omega^{2}=0 .
$$

Thus

$$
\left(r^{2} e^{\alpha S} w^{\prime}(r)\right)^{\prime}+r^{2}\left(4 \pi G \rho-2 \Omega^{2}\right)=0,
$$


where $G$ is the Newtonian gravitational constant. Therefore

$$
r^{2} e^{\alpha S} w^{\prime}(r)=\frac{2 r^{3} \Omega^{2}}{3}-G M(r)
$$

for $r \leq R(p, \sigma)$. By the proofs of part (2) and (3) of Theorem 2.1, we have $w^{\prime}(r) \leq 0$ for $r \leq R(p, \sigma)$. Thus $M(r) \geq \frac{2 r^{3} \Omega^{2}}{3 G}$ for $r \leq R(p, \sigma)$. The estimate (2.23) follows immediately from (2.22).

\section{Proofs of Theorems 2.2 and 2.3}

In this section, we consider the isentropic case $S=$ constant. Theorem 2.1 can be applied in this case because the conditions imposed on the entropy $S$ in Theorem 2.1 are automatically satisfied when $S$ is constant. Without loss of generality, throughout this section, we assume

$$
S=0
$$

for convenience. We prove Theorems 2.2 and 2.3 as follows. First, we define

$$
f(w)=w^{q}-\sigma, \quad w \geq 0,
$$

and

$$
F(w)=\frac{w^{q+1}}{q+1}-\sigma w, \quad w \geq 0 .
$$

We still use $w(r, p, \sigma)$ to denote the solution of the problem

$$
w^{\prime \prime}(r)+\frac{2}{r} w^{\prime}(r)+f(w)=0,
$$

and

$$
w^{\prime}(0)=0, w(0)=p>0 .
$$

Let $R(p, \sigma)$ be defined as in (2.13); $R(p, \sigma)$ is the first point at which $w$ is 0 . Then we have the following proposition.

Proposition 4.1. If $\sigma>0$ and $1<q<5(6 / 5<\gamma<2)$, then

$$
R(p, \sigma)=+\infty, \text { if } 0 \leq p<\underline{p}
$$

where

$$
\underline{p}=\left(\frac{5 \sigma(1+q)}{5-q}\right)^{1 / q}=\left(\frac{10 \gamma \Omega^{2}}{5 \gamma-6}\right)^{\gamma-1} .
$$


Proof. It is easy to verify that $\left(\frac{5 \sigma(1+q)}{5-q}\right)^{1 / q}$ is the unique positive zero of $F(w)-$ $\frac{f(w) w}{6}$. If $R(p, \sigma)<+\infty$, we let $R(p, \sigma)=R>0$. Then $G(R) \geq 0$, where the function $G$ is defined as in (2.5) with $S=0$. On the other hand, $0 \leq w(r) \leq p$ for $0 \leq r \leq R$. If $p<\underline{p}=\left(\frac{5 \sigma(1+q)}{5-q}\right)^{1 / q}$, then $\left(F(w)-\frac{f(w) w}{6}\right)(r)<0$ for $0 \leq r \leq R$. Hence, (3.8) implies $G(R)<0$. This is a contradiction.

First we prove part (1) of Theorem 2.2. For fixed $\sigma>0$, let

$$
p_{0}=\inf \{p: R(p, \sigma)<+\infty\}
$$

By part (3) of Theorem 2.1 and Proposition 4.1, we know that

$$
\underline{p} \leq p_{0}<+\infty
$$

where $p$ is given by (4.7). If we can show

$$
R\left(p_{0}, \sigma\right)<\infty
$$

then part (1) in Theorem 2.2 will be proved. Now (4.9) can be shown by the following argument. Write (4.4) as a first-order system

$$
w^{\prime}=v, \quad v^{\prime}=-\frac{2}{r} v+\sigma-w^{q}
$$

with initial condition

$$
w(0)=p_{0}>0, v(0)=0 .
$$

Define the Hamiltonian $H(w, v)$ by

$$
H(w, v)=\frac{v^{2}}{2}+\frac{w^{q+1}}{q+1}-\sigma w .
$$

Then $H^{\prime}=-\frac{2}{r} v^{2}$, so that $H$ decreases on orbits of (4.10). This implies that the solution of (4.10), (4.11) must have $v$ bounded from below in the region $S=\{w \geq 0$, $v \leq 0\}$. This solution also cannot exit $S$ via $w=0$ at some $v<0$ for some $R>0$; otherwise, since $v^{\prime}(R)=-\frac{2}{R} v(R)+\sigma>0$, the solution crosses the line $w=0$ transversally, so by continuity, there would be a neighborhood $N$ of $p_{0}$ on the $w$ axis, such that for $p \in N$, the orbit of (4.10) satisfying $w(0)=p, v(0)=0$, would also exit $S$ at a point near $w=0, v(R)<0$. This would contradict the definition of $p_{0}$. Similarly the $p_{0}$ orbit cannot exit $S$ via some point $(w, 0)$, with $0<w<p_{0}$. Thus the $p_{0}$ orbit exits $S$ via $w=0, v=0$, so (2.31) holds, and this proves part (1) of Theorem 2.2.

Remark 7. It is not hard to show that every solution of (4.10) satisfying $w(0)=p$, $v(0)=0$, tends to the rest point $\left(w=\sigma^{1 / q}, v=0\right)$ as $r \rightarrow \infty$. 
Now we prove part (2) of Theorem 2.2. First, we show that the radius of the star increases with the increasing angular velocity. We use $w_{i}(r)(i=1,2)$ to denote the solution to the initial-value problem

$$
\begin{aligned}
& w_{i}^{\prime \prime}(r)+\frac{2}{r} w_{i}^{\prime}(r)+w_{i}^{q}-\sigma_{i}=0, \quad r>0, \\
& w_{i}(0)=p, w_{i}^{\prime}(0)=0 .
\end{aligned}
$$

We assume

$$
\sigma_{1}>\sigma_{2}>0
$$

and we want to show that

$$
R\left(p, \sigma_{1}\right) \geq R\left(p, \sigma_{2}\right) .
$$

(Note that each of these are finite, by what we have already shown in part (1) of the theorem).

From (4.13), we have

$$
w_{i}^{\prime}(r)=\frac{1}{r^{2}} \int_{0}^{r} s^{2}\left(\sigma_{i}-w_{i}^{q}(s)\right) d s, \quad i=1,2,
$$

It is easy to verify, using L'Hospital's rule, that

$$
\lim _{r \rightarrow 0+} \frac{2}{r} w_{i}^{\prime}(r)=\frac{2}{3}\left(\sigma_{i}-p^{q}\right) .
$$

It follows from (4.13) and (4.17) that

$$
\lim _{r \rightarrow 0+} w_{i}^{\prime \prime}(r)=-\left(p^{q}-\sigma_{i}\right) / 3 .
$$

Since $\sigma_{1}>\sigma_{2}$, (4.18) implies

$$
w^{\prime \prime} 1(0+)>w_{2}^{\prime \prime}(0+) .
$$

This, together with the fact $w_{1}(0)=w_{2}(0)=p$ and $w^{\prime}(0)=w_{2}^{\prime}(0)=0$, leads to

$$
w_{1}(r)>w_{2}(r) \quad \text { for small } r>0 .
$$

We shall show (4.15) by contradiction. If (4.15) were false, then there would exist an $r_{0} 0<r_{0}<R\left(p, \sigma_{1}\right)<R\left(p, \sigma_{2}\right)$ such that

$$
w_{1}(r)>w_{2}(r) \text { for } 0<r<r_{0} \text {, and } w_{1}\left(r_{0}\right)=w_{2}\left(r_{0}\right) .
$$

Let

$$
y(r)=w_{1}(r)-w_{2}(r) .
$$

We then have, from (4.13),

$$
y^{\prime \prime}(r)+\frac{2}{r} y^{\prime}(r)+y B(r)-\left(\sigma_{1}-\sigma_{2}\right)=0 .
$$


Here

$$
B(r)=q \int_{0}^{1}\left(\lambda w_{1}+(1-\lambda) w_{2}\right)^{q-1}(r) d \lambda .
$$

From (4.16) and (4.21), we have

$$
y(0)=y^{\prime}(0)=0, y(r)>0 \text { for } 0<r<r_{0} \text {, and } y\left(r_{0}\right)=0 .
$$

Multiplying (4.22) by $y^{\prime}$ and integrating the resulting equation over the interval $\left[0, r_{0}\right]$, we get, since $y^{\prime}(0)=0$,

$$
\begin{aligned}
& \frac{\left(y^{\prime}\left(r_{0}\right)\right)^{2}}{2}+\int_{0}^{r_{0}} \frac{2}{r}\left(y^{\prime}(r)\right)^{2} d r \\
& \quad+\int_{0}^{r_{0}} B(r) y y^{\prime}(r) d r-\int_{0}^{r_{0}}\left(\sigma_{1}-\sigma_{2}\right) y^{\prime}(r) d r=0 .
\end{aligned}
$$

With the help of (4.24) and integration by parts, we get

$$
\int_{0}^{r_{0}} B(r) y y^{\prime}(r) d r=\int_{0}^{r_{0}} B(r)\left(\frac{y^{2}}{2}\right)^{\prime} d r=-\frac{1}{2} \int_{0}^{r_{0}} B^{\prime}(r) y^{2}(r) d r
$$

and

$$
\int_{0}^{r_{0}}\left(\sigma_{1}-\sigma_{2}\right) y^{\prime}(r) d r=0
$$

Substituting (4.26) and (4.27) into (4.25), we have

$$
\frac{\left(y^{\prime}\left(r_{0}\right)\right)^{2}}{2}+\int_{0}^{r_{0}} \frac{2}{r}\left(y^{\prime}(r)\right)^{2} d r+\frac{1}{2} \int_{0}^{r_{0}}\left(-B^{\prime}(r)\right) y^{2}(r) d r=0 .
$$

By the definition of $B(r)$ (see (4.23)), we have

$$
B^{\prime}(r)=q(q-1) \int_{0}^{1}\left(\lambda w_{1}+(1-\lambda) w_{2}\right)^{q-2}\left(\lambda w_{1}^{\prime}(r)+(1-\lambda) w_{2}^{\prime}(r)\right) d \lambda .
$$

Since $w_{i}^{\prime}(r)<0$ for $0<r<R\left(p, \sigma_{i}\right)(i=1,2)$ (see [9]), we thus have

$$
B^{\prime}(r)<0 \text { for } 0<r \leq r_{0},
$$

when $\gamma<2$, i.e., $q>1$. Hence, each term in (4.28) must be zero. This contradicts (4.24), and proves (4.15). Now we show that the radius of the star decreases with the increasing central density, i.e.,

$$
R\left(p_{1}, \sigma\right) \geq R\left(p_{2}, \sigma\right)
$$

if $p_{0} \leq p_{1}<p_{2}$ and $\sigma>0$. For this purpose, let $w(r, p, \sigma)$ be the solution of the following initial-value problem:

$$
\begin{aligned}
& w^{\prime \prime}(r)+\frac{2}{r} w^{\prime}(r)+w^{q}-\sigma=0, \\
& w(0)=p, w^{\prime}(0)=0
\end{aligned}
$$


for $p \geq p_{0}$. We use the rescaling

$$
\lambda=r^{(q-1) / 2}, \theta(\lambda)=u / p ;
$$

then $\theta(\lambda)$ is the solution of the initial-value problem

$$
\begin{aligned}
& \theta_{\lambda \lambda}+\frac{2}{\lambda} \theta_{\lambda}+\theta^{q}-\frac{\sigma}{p^{q}}=0, \lambda>0, \\
& \theta(0)=1, \theta^{\prime}(0)=0 .
\end{aligned}
$$

The first zero of $\theta$ depends only on the parameter $\frac{\sigma}{p^{q}}$. We use $\lambda\left(\frac{\sigma}{p^{q}}\right)$ to denote this first zero. Then by (4.33), we have

$$
R(p, \sigma)=p^{(1-q) / 2} \lambda\left(\frac{\sigma}{p^{q}}\right)
$$

for $p \geq p_{0}$. Similar to the argument in the proof of (4.15), we can show that $\lambda\left(\frac{\sigma}{p^{q}}\right)$ increases with the parameter $\frac{\sigma}{p^{q}}$. Thus, for the fixed $\sigma$, it decreases with $p$ for $p \geq p_{0}$. This, together with (4.34) and the fact $q>1$, implies (4.31). This completes the proof of part (2) of Theorem 2.2.

Remark 8. The above scaling argument also works for a non-rotating star, i.e., the case when $\sigma=0$. For the non-rotating star, the radius of the star, $R(p, 0)$, is always finite for $p>0$ if $6 / 5<\gamma<2$ (see [5]). For the non-rotating star, (4.34) becomes

$$
R(p, 0)=\lambda_{0} p^{(1-q) / 2}, \quad q=\frac{1}{\gamma-1},
$$

where $\lambda_{0}$ is the first zero of the function $\theta(\lambda)$, which is the solution of the following initial-value problem:

$$
\begin{gathered}
\theta_{\lambda \lambda}+\frac{2}{\lambda} \theta_{\lambda}+\theta^{q}=0, \lambda>0, \\
\theta(0)=1, \theta^{\prime}(0)=0 .
\end{gathered}
$$

From (4.35) we see that the radius of a non-rotating star is proportional to $p^{(1-q) / 2}$.

We now turn to the proof of Theorem 2.3. In (4.34), since $\lambda\left(\frac{\sigma}{p^{q}}\right)$ increases with the parameter $\frac{\sigma}{p^{q}}$, as we have already shown, we have

$$
\lambda\left(\frac{\sigma}{p^{q}}\right) \leq \lambda\left(\frac{\sigma}{p_{0}^{q}}\right),
$$

for $p \geq p_{0}$. We apply (4.34) to the case of $p=p_{0}$ and obtain

$$
R\left(p_{0}, \sigma\right)=p_{0}^{(1-q) / 2} \lambda\left(\frac{\sigma}{p_{0}^{q}}\right) .
$$

Now $R\left(p_{0}, \sigma\right)<+\infty$, from part (2) of Theorem 2.1, and $p_{0}$ is a positive constant determined completely by $\gamma$ and $\sigma$. Thus $\lambda\left(\frac{\sigma}{p_{0}^{q}}\right)$ is a positive constant determined also only by $\gamma$ and $\sigma$. We set this positive constant as $C$ in (4.36), then (2.31) follows. Since $\gamma<2$, (2.31) implies (2.32). The proof of Theorem 2.3 is complete. 
The following lemma will be useful in proving Theorem 2.4.

Lemma 4.2. For any $\sigma>0$, there exists $R_{0}>0$ such that (2.25) with the data $w^{\prime}(0)=0$ and $w(R)=0$ has a positive solution in the ball $B_{R}(0)$ for all $R \leq R_{0}$, if $1<q<5(6 / 5<\gamma<2)$.

Proof. Fix $\sigma>0$, and write $R(p, \sigma)=R(p)$. By Theorem 2.2 Part (2), there exists $p_{0}>0$ such that $R(p)<+\infty$ for any $p \geq p_{0}$. Let $R_{0}=R\left(p_{0}\right)$. For any $0<R<R_{0}$, it follows from (2.32) that there exits $p_{1}>p_{0}$ such that $R\left(p_{1}\right)<R$. Now $R\left(p_{0}\right)>R>R\left(p_{1}\right)$, and $R(p)$ is a continuous function of $p$ (actually, it is differentiable; cf. [17]). Thus, by the intermediate-value theorem, there exists $p \in\left(p_{0}, p_{1}\right)$ such that $R(p)=R$.

\section{Proofs of Theorems $2.4,2.5$ and 2.6}

In this section, we consider the case for the general domains and variable angular velocity. In Theorems 2.4 and 2.5, we assume the entropy function $S(x)$ is constant. We set $S=0$ for convenience. Substituting this in (2.5), we get

$$
\Delta w+w^{q}-2 \Omega(\eta)\left(\Omega(\eta)+\eta \Omega^{\prime}(\eta)\right)=0
$$

We look for the solution of (5.1) satisfying the condition

$$
w(x)>0, x \in D, w(x)=0, x \in \partial D .
$$

In order to prove Theorem 2.4, we need a comparison lemma, which can be found in [7] or [16] . Before we state this lemma, we give the following definitions of weak sub- and supersolutions of the problem (5.1) and (5.2). For notational convenience, we define the function $f(x, w)$ by

$$
f(x, w)=w^{q}-2 \Omega(\eta(x))\left[\Omega(\eta(x))+\eta(x) \Omega^{\prime}(\eta(x)] .\right.
$$

Definition. (a) A function $\bar{w} \in H^{1}(D)$ is called a weak supersolution of problem (5.1) and (5.2) if

$$
\int_{D} \nabla \bar{w} \cdot \nabla v d x \geq \int_{D} f(x, \bar{w}) v d x
$$

for each $v \in C_{0}^{1}(D), v \geq 0$ a.e., where the subscript zero denotes $v=0$ on $\partial D$.

(b) Similarly $\underline{w} \in \bar{H}^{1}(D)$ is called a weak subsolution of problem (5.1) and (5.2) if

$$
\int_{D} \nabla \underline{w} \cdot \nabla v d x \leq \int_{D} f(x, \underline{w}) v d x
$$

for each $v \in C_{0}^{1}(D), v \geq 0$ 
The following lemma is well known; cf. [7] or [16].

Lemma 5.1. Assume there exists a weak supersolution $\bar{w}$ and a weak subsolution $\underline{w}$ of (5.1) and (5.2) satisfying

$\underline{w} \leq 0, \bar{w} \geq 0$ on $\partial D$ in the trace sense, and $\underline{w} \leq \bar{w}$ a.e. in $D$.

Then there exists a weak solution $w \in H_{0}^{1}(D)$ of (5.1) and (5.2), such that

$$
\underline{w} \leq w \leq \bar{w} \quad \text { a.e. in D. }
$$

Remark 9. By the smoothness assumptions of $\partial D$ and $\Omega(\eta)$, the usual regularity arguments (see [10]) show that a weak positive solution of (5.1) and (5.2) must be a classical solution.

Let $D$ be a bounded domain with smooth boundary, and assume $\Omega(\eta) \in$ $C^{1+\delta}(D) \cap C(\bar{D})$ for some $\delta, 0<\delta<1$. Define $\beta$ by

$$
\beta=: \max _{x \in \bar{D}} \mid 2 \Omega(\eta)\left(\Omega(\eta)+\eta \Omega^{\prime}(\eta) \mid,\right.
$$

where $\eta=\eta(x)$ is as defined in Section 1. Let $B_{R_{1}}(0)$ be the biggest ball contained in $D$. Consider the positive solution of the equation

$$
u^{\prime \prime}(r)+\frac{2}{r} u^{\prime}(r)+u^{q}-\beta=0,
$$

with data

$$
u^{\prime}(0)=0, u(r)>0 \text { for } 0<r<R_{1}, u\left(R_{1}\right)=0 .
$$

By Lemma 4.2, if $6 / 5<\gamma<2$, then there exists a positive constant $R_{2}$ depending only on $\beta$ and $q$ such that (5.8) and (5.9) has a solution if $0<R_{1} \leq R_{2}$. We require

$$
D \subset B_{R_{2}}(0) .
$$

This implies that (5.8) and (5.9) has a positive solution. We denote this solution by $u(x)=u(|x|)\left(x \in B_{R_{1}}(0)\right)$. A subsolution to (5.1) and (5.2) can now be constructed. First define $\underline{w}(x)$ by

$$
\underline{w}(x)= \begin{cases}u(|x|) & \text { for } x \in B_{R_{1}}(0), \\ 0 & \text { for } x \in D-B_{R_{1}}(0) .\end{cases}
$$

Lemma 5.2. If Condition $A$ in Section 1 holds, then $\underline{w}(x)$ defined by (5.11) is a weak subsolution to (5.1) and (5.2).

Proof. First, by the definition of $\underline{w}$, we have

$$
\begin{aligned}
\int_{D} & (\nabla \underline{w} \cdot \nabla v-f(x, \underline{w}) v) d x \\
= & \int_{B_{R_{1}}(0)}(\nabla u \cdot \nabla v-f(x, u) v) d x \\
& +\int_{D-B_{R_{1}}(0)} 2 \Omega(\eta(x))\left[\Omega(\eta(x))+\eta(x) \Omega^{\prime}(\eta(x)] v(x) d x\right.
\end{aligned}
$$


for each $v \in C_{0}^{1}(D), v \geq 0$. By Condition A in Section 1, we have

$$
\Omega(\eta(x))\left[\Omega(\eta(x))+\eta(x) \Omega^{\prime}(\eta(x)] \leq 0\right.
$$

for $x \in D-B_{R_{1}}(0)$. On the other hand, since $u$ satisfies (5.8) in the ball $B_{R_{1}}(0)$, we have by the divergence theorem,

$$
\begin{aligned}
\int_{B_{R_{1}}(0)} \nabla u \cdot \nabla v d x & =-\int_{B_{R_{1}}(0)} \Delta u v d x+\int_{\partial B_{R_{1}}(0)} \frac{\partial u}{\partial v} v d S \\
& \leq \int_{B_{R_{1}}(0)} f(x, u) v d x+\int_{\partial B_{R_{1}}(0)} \frac{\partial u}{\partial v} v d S
\end{aligned}
$$

where $v$ is the unit outer normal vector. Since $u^{\prime}(R) \leq 0$, we have $\frac{\partial u}{\partial v} \leq 0$ on $\partial B_{R_{1}}(0)$, and since $u(x)=\underline{w}(x)$ for $x \in B_{R_{1}}(0),(5.13)$ implies

$$
\int_{B_{R_{1}}(0)} \nabla u \cdot \nabla v d x \leq \int_{B_{R_{1}}(0)} f(x, \underline{w}) \cdot v d x,
$$

so that

$$
\int_{D}(\nabla \underline{w} \cdot \nabla v-f(x, \underline{w}) v) d x \leq 0
$$

and this proves Lemma 5.2.

We next construct a supersolution to (5.1) and (5.2). Let $R_{2}$ be the positive number as in (5.10) such that $D \subset B_{R_{2}}(0)$ and $\bar{p}$ be any positive number such that

$$
\bar{p}>\max _{x \in \bar{D}} \underline{w}(x),
$$

where $\underline{w}$ is the subsolution which we have already constructed. We consider the following boundary-value problem:

$$
\Delta \bar{w}+\bar{w}^{q}+\beta=0 \text { for } x \in B_{R_{2}}(0),
$$

and

$$
\left.\bar{w}\right|_{\partial B_{R_{2}}(0)}=\bar{p} .
$$

By a result in [12], there exists a unique positive solution $\tilde{w}(x)$ to problem (5.18) and (5.19). Furthermore, by the maximum principle, we have

$$
\bar{w}(x) \geq \bar{p} \quad \text { for } x \in B_{R_{2}}(0) .
$$

Therefore, by the choice of $\bar{p}$ (see (5.17)), we have

$$
\bar{w}(x) \geq \underline{w}(x) \quad \text { for } x \in \bar{D} .
$$

It is easy to verify that $\bar{w}$ satisfies (5.3), i.e., $\bar{w}(x)$ is a supersolution of (5.1) and (5.2). Then (5.5) follows from (5.21) and the properties of $\underline{w}$ and $\bar{w}$. Hence, by Lemma 4.1, problem (5.1) and (5.2) has a solution $w$ satisfying $\underline{w}(x) \leq w(x) \leq \bar{w}(x)$ for 
$x \in D$. Inequality (2.34) can be proved as (2.28). Now we prove $w(x)>0$ for $x \in D$. Since $w(x) \geq \underline{w}(x)$ for $x \in D$, and $\underline{w}(x)>0$ if $x \in B_{R_{1}}(0)$, it suffices to show $w(x)>0$ as $x \in D-B_{R_{1}}(0)$. This can be proved by the strong maximum principle (see [10]), using Condition A in Section 1. This completes the proof of Theorem 2.4.

We now prove Theorem 2.5. For this, we recall that the solution $w(x)$ of (5.1) and (5.2) is constructed by the following iteration (cf. [7] or [16]). Set $w_{0}(x)=\underline{w}(x)$, and then define $w_{k}(k=0,1,2, \ldots)$ inductively to be the unique weak solution of the linear boundary-value problem

$$
\begin{aligned}
-\Delta w_{k+1}+C w_{k+1} & =f\left(w_{k}\right)+C w_{k} \quad \text { in } D, \\
w_{k+1} & =0 \quad \text { on } \partial D,
\end{aligned}
$$

where $C=\max _{x \in \bar{D}}\left|f^{\prime}(\bar{w})\right|(x)$. Then it can be shown (cf. [16])

$$
\underline{w}=w_{0} \leq w_{1} \leq \cdots \leq w_{k} \leq \cdots \leq \bar{w}, \text { a.e. in } D .
$$

Set $w(x)=\lim _{k \rightarrow \infty} w_{k}(x)$; then $w(x)$ is the desired solution. Since $\underline{w}$ and $\bar{w}$ are axi-symmetric, if $D$ is an axi-symmetric domain, it is not hard to show each $w_{k}(k=0,1,2, \ldots)$ is axi-symmetric; i.e., depends only on $\eta$ and $z$. Indeed, if $\eta=\sqrt{x_{1}^{2}+x_{2}^{2}}$, and $z=x_{3}$, then the Laplacian transforms to

$$
\partial_{\eta \eta}+\frac{1}{\eta} \partial_{\eta}+\partial_{z z}
$$

So $w_{1}$ satisfies the equation

$$
-\left(\partial_{\eta \eta} w_{1}+\frac{1}{\eta} \partial_{\eta} w_{1}+\partial_{z z} w_{1}\right)+C w_{1}=f(\underline{w})+C \underline{w},
$$

together with the boundary condition $w_{1}=0$ on $\partial D$. Since the right-hand side of (5.22) depends only on $\eta$ and $z$, and since this Dirichlet problem, being linear, has a unique solution, it follows that $w_{1}$ is axi-symmetric. Similarly, each $w_{k}$ is axi-symmetric. Thus $w(x)$ is axi-symmetric. This completes the proof of Theorem 2.5 .

We finally prove Theorem 2.6; the proof makes use of the celebrated POHOZAEV identity (see [14]).

Multiply (2.5) by $x \cdot \nabla w$ and $w$ respectively, and integrate the resulting equations over $D$. After some manipulation (details can be found in [14]), we obtain the following "Pohozaev-like" identity

$$
\begin{gathered}
\int_{D} K\left(\frac{1}{2}-\frac{3}{q+1}\right) w^{q+1} d x+\int_{D}\left[\frac{1}{2} e^{\alpha S}|\nabla w|^{2}+\frac{\alpha K e^{-\alpha S}}{q+1} w^{q+1}\right](x \cdot \nabla S) d x \\
+\int_{D}\left(\eta A^{\prime}(\eta)+\frac{5 A(\eta)}{2}\right) w d x+\int_{\partial D} \frac{1}{2} e^{\alpha S}|\nabla w|^{2}(x \cdot v) d S=0
\end{gathered}
$$

where $A(\eta)=2 \Omega(\eta) \Omega(\eta)+\eta \Omega^{\prime}(\eta)$, and $v$ is the unit outer normal vector. If $\gamma \leq 6 / 5$ (or $\gamma<6 / 5$ ), then $q \geq 5$ (or $q<5$ ) and thus $\frac{1}{2}-\frac{3}{q+1} \geq 0$ (resp. 
$\left.\frac{1}{2}-\frac{3}{q+1}>0\right)$. Thus, if $\eta A^{\prime}(\eta)+\frac{5 A(\eta)}{2}>0$ (or $\left.\eta A^{\prime}(\eta)+\frac{5 A(\eta)}{2} \geq 0\right), x \cdot v \geq 0$ and $x \cdot \nabla S \geq 0,(5.23)$ implies $w(x)=0$ for $x \in D$. This completes the proof of Theorem 2.6.

\section{Further discussion}

In this section, we give further discussions on the difference between our formulation of the problem and that in [2] and [11]. As mentioned in Section 1, for the isentropic case, i.e., $S=$ constant, system (1.3) is considered in [2] and [11] in all of $R^{3}$ space, with the prescribed total mass M. In [2] and [11], the function $\Phi$ is given by

$$
\Phi(x)=-G \int_{R^{3}} \frac{\rho(y)}{|x-y|} d y .
$$

This choice is natural when we consider the equation $\Delta \Phi=4 \pi G \rho$ in all of $R^{3}$, because any bounded solution of $\Phi$ for this equation in $R^{3}$ differs from (6.1) only by a constant.

In our formulation, we consider system (1.3) in a bounded domain $D$, the domain of a star. The boundary condition in our formulation is $\rho=0$, to match the exterior vacuum continuously. In this formulation, the function $\Phi(x)$ could be different from the formula

$$
\Phi(x)=-G \int_{D} \frac{\rho(y)}{|x-y|} d y,
$$

due to the boundary effects. We illustrate this by considering the isentropic case, i.e., $S=$ constant (without loss of generality, $S$ is assumed to be zero). In this case, the first equation in (1.3) reduces to

$$
\nabla(w+\Phi-J)=0,
$$

where $w$ is given by (2.3) (with $S=0$ ) $J$ is given by (1.4). From (6.3), we have

$$
w+\Phi-J=C,
$$

where $C$ is a constant. On the boundary $\partial D$, since $\gamma>1$, the boundary condition $\rho=0$ implies $w=0$ on the boundary $\partial D$. This, together with (6.4), implies that

$$
\Phi(x)=C+J \quad \text { for } x \in \partial D .
$$

The solution of the problem $\Delta \Phi=4 \pi G \rho$ with the boundary condition (6.5) could be different from formula (6.2). The difference is a harmonic function which counts the boundary effects.

When the domain $D$ is a ball and the entropy $S$ is a constant, we give the existence results and study some physical properties of the solutions. Historically, incompressible-fluid models of rotating stars are studied extensively. For the incompressible-fluid model, density is a positive constant inside a star and zero outside the star, so there is a discontinuity of density across the boundary of a star. The problem 
for the incompressible-fluid model of Newtonian rotating stars is to determine the boundaries of stars with the prescribed angular velocity (or angular momentum) and total mass. For such problems, explicit solutions are found (e.g. Maclaurin spheroids and Jacobi ellipsoids). Those spheroids and ellipsoids are close to balls if the angular velocities are small. For the compressible-fluid model, the problem is quite different since the distribution of density must be determined. For the compressible-fluid model of a rotating star, if the domain $D$ is not a ball, it seems very difficult to obtain any interesting properties of solutions like those we obtain in Theorems $2.1-2.3$ for the case when $D$ is a ball. When $D$ slightly differs from a ball, for example, an ellipsoid with eccentricity close to one, it is reasonable to expect the solutions obtained in this paper, for the case when $D$ is a ball, to give good approximations.

Some non-existence results are proved in [11] for large angular velocity $\Omega$ for the problem formulated in [2]. This means, in order to ensure existence of solutions for the problem formulated in [2], that the angular velocity cannot exceed a critical value. This critical value is given in term of total mass $M$, which is prescribed in the formulation of the problem in [2] and [11]. Our existence theorem, Theorem 2.1 , is valid for any angular velocity $\Omega$. This difference is due to the different formulations of problems mentioned above. In our formulation, we do not prescribe the total mass, instead we prescribe the domain. The total mass thus depends on the angular velocity and the domain. We also give a lower bound of the total mass (cf. (2.22)) in terms of angular velocity.

Acknowledgements. Part of this work was completed when TAO LUO was an assistant professor at the University of Michigan. JOEL SMOLLER was supported in part by the NSF, contract number DMS-010-3998. We are grateful to the referee for his very interesting remarks and comments, which enabled a new section, Section 6, to be added in the final version of the paper.

\section{References}

1. AdLER, R. BAZIN, M., SCHIFFER, M.: Introduction to General Relativity. McGraw-Hill, New York (1975)

2. Auchmuty, G., BeALS, R.: Variational solutions of some nonlinear free boundary problems. Arch. Rational Mech. Anal. 43, 255-271 (1971)

3. Caffarelli, L., Friedman, A.: The shape of axi-symmetric rotating fluid. J. Funct. Anal. 694, 109-142 (1980)

4. CASTRO, A., SHIVAJI, R.: Non-negative solutions for a class of radially symmetric nonpositone problems. Proc. AMS 106, 735-740 (1989)

5. CHANDRASEKHAR, S.: Introduction to the Stellar Structure. University of Chicago Press (1939)

6. Chanillo, S., LI, Y.Y.: On diameters of uniformly rotating stars. Comm. Math. Phys. 166, 417-430 (1994)

7. Evans, L.: Partial Differential Equations. Graduate Studies in Math., AMS, Providence, Rhode Island (1998)

8. DENG, Y., LiU, T.P., YANG, T., YAO, Z.: Solutions of Euler-Poisson equations for gaseous stars. Arch. Rational Mech. Anal. 164, 261-285 (2002)

9. GIDAS, B., NI, W., NiRENBERG, L.: Symmetry of positive solutions of nonlinear elliptic equations in $R^{n}$. Comm. Math. Phys. 68, 209-243 (1975) 
10. Gilbarg, D., Trudinger, N.: Elliptic Partial Differentail Equations of Second Order (2nd ed.) Springer (1983)

11. LI, Y. Y.: On uniformly rotating stars. Arch. Rational Mech. Anal. 115, 367-393 (1991)

12. LIONS, P. L.: On the existence of positive solutions of semilinear elliptic equations. SIAM, Rev. 24, 441-467 (1982)

13. MAKINO, T.: Blowing up of the Euler-Poisson equation for the evolution of gaseous star. Transport Theory and Statistical Physics 21, 615-624 (1992)

14. PoHOzAEV, S. I.: Eigenfunctions of the equations $\Delta u+\lambda f(u)=0$. Sov. Math. Dok. 5, 1408-1411 (1965)

15. RABINOWITZ, P.: Minimax methods in critical point theory with applications to differential equations. CBMS \# 65, AMS (1986)

16. SMOller, J.: Shock Waves and Reaction-Diffusion Equations. Springer, Berlin, New York (1983)

17. Smoller, J., Wasserman, A.: Existence of positive solutions for semilinear elliptic equations in general domains. Arch. Rational Mech. Anal. 98, 229-249 (1987)

18. Weinberg, S.: Gravitation and Cosmology. John Wiley and Sons, New York, (1972)

Department of Mathematics,

Georgetown University,

Washington DC 20057-1233.

USA

e-mail: t148@georgetown.edu

and

Department of Mathematics,

University of Michigan

525 East University Ave.

Ann Arbor, MI 48109-1109.

USA

e-mail: smoller@umich.edu

(Accepted February 16, 2004)

Published online May 4, 2004 - (C) Springer-Verlag (2004) 